\title{
Dietas vegetarianas e desempenho esportivo
}

\section{Vegetarian diets and sports performance}

Lucas Guimarães FERREIRA ${ }^{1}$

Roberto Carlos BURINI2

Adriano Fortes MAIA ${ }^{1,3}$

RE S U M O

As evidências atuais apontam benefícios da dieta vegetariana para a saúde humana. Contudo, a partir da adoção de práticas vegetarianas mais restritivas, confirmam-se os riscos à saúde. As dietas vegetarianas são caracterizadas pelo elevado consumo de carboidratos, fibras, magnésio, potássio, folato e antioxidantes, podendo apresentar deficiências em aminoácidos e ácidos graxos essenciais, cálcio, zinco, ferro e cobalamina. Pesquisas experimentais em humanos indicam que vegetarianos e não-vegetarianos apresentam capacidade aeróbica semelhante. Em relação ao desempenho em atividades de força e potência muscular, as pesquisas são escassas, mas as existentes não apontam diferenças significativas. Situações de risco cardiovascular têm sido confirmadas, devido ao provável quadro de hiperhomocisteinemia, em decorrência da baixa ingestão de cobalamina. As dietas vegetarianas são isentas de creatina, o que resulta em estoques musculares mais baixos nessa população. Possivelmente ocorrem alterações hormonais e metabólicas em resposta às dietas vegetarianas, como baixos níveis de testosterona e androstenediona. A função imune parece não ser prejudicada. Dessa forma, a prática de dietas vegetarianas apresenta-se compatível com a prática esportiva cotidiana, desde que bem planejada para evitar deficiências nutricionais.

Termos de indexação: creatina; exercício; dieta vegetariana.

\section{A B S T R A C T}

Current evidences show benefits of a vegetarian diet for human health. However, when a stricter vegetarian diet is adopted, health risks are confirmed. Vegetarian diets are characterized by a high intake of carbohydrates, fibers, magnesium, potassium, folate and antioxidants and may result in a low intake of amino acids, essential fatty acids, calcium, zinc, iron and cobalamin. Experimental human researches indicate that both vegetarians and non-vegetarians present similar aerobic capacity. Regarding muscular strength and power, researches are

\footnotetext{
1 Grupo de Estudos e Pesquisas em Nutrição e Metabolismo do Exercício, Centro de Educação Física e Desportos, Universidade Federal do Espírito Santo. Vitória, ES, Brasil.

${ }^{2}$ Centro de Metabolismo em Exercício e Nutrição, Faculdade de Medicina de Botucatu, Universidade Estadual Paulista. Botucatu, SP, Brasil.

3 Departamento de Desportos, Centro de Educação Física e Desportos, Universidade Federal do Espírito Santo. Av. Fernando Ferrari, 514, Goiabeiras, 29075-910, Vitória, ES, Brasil. Correspondência para/Correspondence to: A.F. MAIA. E-mail: <adriano@cefd.ufes.br>
} 
scarce but the existent ones do not report significant differences. Cardiovascular risk situations have been confirmed, due to the possible hyperhomocysteinemia given the low ingestion of cobalamin. Vegetarian diets do not contain creatine, resulting in lower muscle reserves of this nutrient among this population. Hormonal and metabolic changes are a possibility in response to vegetarian diets, as well as low levels of testosterone and androstenedione. The immune function does not seem to be affected. Thus, a vegetarian diet is compatible with daily exercising as long as it is well planned in order to avoid nutritional deficiencies.

Indexing terms: creatine; exercise; diet, vegetarian.

\section{N T R O D U Ç Ã O}

O vegetarianismo abrange ampla variedade de práticas dietéticas com diferentes implicações para a saúde ${ }^{1}$. Assim, há diferentes definições e fundamentações das práticas vegetarianas, como, por exemplo: a dieta vegan, que não contém nenhum produto de origem animal; a lactoovovegetariana (LOV), que inclui produtos lácteos e ovos; e a semivegetariana, que ainda inclui pequenas quantidades ou consumo esporádico de determinados tipos de carne, como peixes e aves ${ }^{2}$. As dietas não-vegetarianas, nas quais o consumo de carne é freqüente, são denominadas dietas onívoras.

A adoção de dieta vegetariana tem sido associada a diversos benefícios para a saúde da população humana, como baixas concentrações de lipídios séricos, baixos níveis de adiposidade corporal e baixa incidência de mortes por isquemia do miocárdio, diabetes mellitus e certos tipos de câncer-5, além de uma maior expectativa de vida ${ }^{6}$. Entretanto, também tem sido objeto de preocupação, na medida em que novos estudos realçam possíveis aspectos prejudiciais à saúde com a prática do vegetarianismo. De acordo com a American Dietetic Association ${ }^{7}$, dietas vegetarianas oferecem determinados benefícios nutricionais, como a baixa ingestão de gordura saturada e colesterol - ou mesmo a não elevação desses marcadores, quando observados em relação ao envelhecimento ${ }^{8}$ - a alta ingestão de carboidratos, fibras dietéticas, magnésio, potássio, folato, antioxidantes (como as vitaminas C e E) e fitoquímicos. Em contrapartida, Sabate ${ }^{9}$ cita que a dieta vegetariana desbalanceada ou restritiva, particularmente em situações de altas demandas metabólicas (como durante o exercício), pode provocar deficiências nutricionais.

Algumas pesquisas experimentais foram realizadas no intuito de investigar a relação entre a dieta vegetariana e o desempenho esportivo. Discute-se se tal dieta pode afetar, positiva ou negativamente, o desempenho de atletas de resistência e força.

Para a realização deste artigo, foram coletadas todas as pesquisas disponíveis no banco de dados Medline, que tinham por objetivo verificar a influência das dietas vegetarianas sobre variáveis do desempenho atlético, como a capacidade aeróbica e de força muscular. No total, foram analisadas onze pesquisas experimentais publicadas em periódicos internacionais, disponíveis nesse banco de dados. Com base nos resultados encontrados, discutiram-se as necessidades e carências nutricionais dos atletas vegetarianos, assim como alterações fisiológicas em resposta à dieta e suas possíveis implicações no desempenho atlético.

\section{Vegetarianismo e desempenho}

Observaram-se, na literatura, diversos estudos avaliando a influência das dietas vegetarianas sobre o desempenho de esportes de endurance e força. Um resumo dos resultados das pesquisas analisadas encontra-se no Quadro 1.

$\mathrm{O}$ volume de pesquisas que testaram a capacidade aeróbica é superior ao daquelas que testaram a força muscular dos vegetarianos. No experimento de Cotes et al. ${ }^{10}$, não foram encontradas diferenças na função pulmonar e na resposta cardiorrespiratória no exercício submá- 
Quadro 1. Resumo dos resultados das pesquisas experimentais analisadas.

\begin{tabular}{lccccc}
\hline Autores & Ano & População & Atividade ${ }^{(1)}$ & Dieta $^{(2)}$ & Resultados $^{(3)}$ \\
\hline Cotes et al. ${ }^{10}$ & 1970 & Adultos & END & VEG & Sem diferença significativa \\
Hanne et al. $^{11}$ & 1986 & Adultos & END e FOR & LOV & Sem diferença significativa \\
Nieman et al. ${ }^{12}$ & 1988 & Adultos & END & LOV & Sem diferença significativa \\
Nieman et al. ${ }^{13}$ & 1989 & Adultos & END & LOV & Sem diferença significativa \\
Snyder et al. ${ }^{14}$ & 1989 & Adultos & END & SEV & Sem diferença significativa \\
Richter et al. ${ }^{15}$ & 1991 & Adultos & END & LOV & Sem diferença significativa \\
Raben et al. ${ }^{16}$ & 1992 & Adultos & END & LOV & Sem diferença significativa \\
Eisinger et al. ${ }^{17}$ & 1994 & Adultos & END & LOV & Sem diferença significativa \\
Campbell et al. ${ }^{19}$ & 1999 & Idosos & FOR & LOV & Sem diferença significativa \\
Hebbelinck et al. ${ }^{18}$ & 1999 & Jovens e Adultos & END e FOR & LOV & Grupo LOV com maior capacidade \\
& & & & aeróbica e menor força muscular ${ }^{(4)}$ \\
\hline
\end{tabular}

(1) END: Resistência Aeróbica (Endurance); FOR: Força; (2) Tipo de dieta vegetariana; LOV: Lactoovovegetariana; VEG: Vegan; SEV: Semi-vegetariana; ${ }^{(3)}$ Referentes aos resultados em testes de força e/ou capacidade aeróbica; ${ }^{(4)}$ Em alguns dos grupos analisados. Ver texto para mais detalhes.

ximo em ciclo-ergômetro, entre vegans e não-vegetarianos. Hanne et al. ${ }^{11}$ também não encontraram diferenças no desempenho de atletas israelenses de ambos os sexos. Diversas outras pesquisas encontraram resultados semelhantes $^{12-18}$.

Campbell et al. ${ }^{19}$ não encontraram diferenças significativas na força muscular dinâmica entre grupos de homens vegetarianos e não-vegetarianos. Ambos os grupos demonstraram aumento similar na força, após 12 semanas de treinamento contra resistência. Esses resultados estão de acordo com os encontrados por Hanne et al. ${ }^{11}$, em pesquisa anterior.

Dos dez estudos analisados, apenas um encontrou diferenças na capacidade aeróbica e em testes de potência ${ }^{18}$. Crianças, adolescentes e adultos vegetarianos foram testados. Os adolescentes e adultos mostraram melhores resultados no teste cardiorrespiratório, mas os adolescentes obtiveram valores mais baixos nos testes de força e potência. Esse experimento não contou com grupo controle não-vegetariano, sendo os resultados comparados a valores de referência indicados por pesquisas anteriores. Talvez a falta de grupo controle possa ter ocasionado resultados divergentes dos demais estudos citados.
Com base nesses resultados, conclui-se que a capacidade aeróbica não parece ser afetada pela adoção de uma dieta vegetariana, desde que esta atenda às necessidades nutricionais do atleta. Dessa forma, uma dieta vegetariana variada e balanceada parece compatível com o desempenho atlético vencedor em modalidades esportivas com predominância no sistema oxidativo. Com relação à força muscular são necessários maiores estudos, dada pouca produção científica que investigou essa questão.

\section{Necessidades nutricionais}

\section{Energia}

De acordo com o posicionamento do American College of Sports Medicine (ACSM) ${ }^{20}$, a baixa ingestão energética pode resultar em perda de massa muscular, distúrbios no ciclo menstrual das atletas, perda de massa óssea e aumento do risco de desenvolverem fadiga e lesões. Ainda segundo o ACSM, a ingestão energética de atletas de endurance deve ser de 3000 a $5000 \mathrm{kcal} / \mathrm{dia}$. Atletas de força usualmente necessitam, minimamente, de $50 \mathrm{kcal} / \mathrm{kg} / \mathrm{dia}$, que representa $3500 \mathrm{kcal} /$ dia para um indivíduo de $70 \mathrm{~kg}$. Segundo Williams ${ }^{21}$, a deficiência energética não representa grande preocupação para os 
vegetarianos, mas é necessária atenção especial para que se alcance uma ingestão adequada de energia, caso contrário o desempenho pode ser prejudicado.

Na pesquisa de Eisinger et al. ${ }^{17}$, atletas lactoovovegetarianos e não-vegetarianos, consumindo uma dieta de $4500 \mathrm{kcal}(60 \%$ advindas de carboidratos, 30\% de proteínas e $10 \%$ de gorduras), não mostraram diferenças na performance em uma maratona. Isso confirma que, desde que as necessidades energéticas sejam alcançadas, independentemente do tipo de dieta, o rendimento não é afetado.

\section{Macronutrientes}

Atletas de endurance necessitam de dieta rica em carboidratos para otimizar os estoques de glicogênio muscular e hepático. Para esses indivíduos, a ingestão diária de carboidratos deve ficar na faixa de $500 \mathrm{~g}$ a $800 \mathrm{~g}$ (8 a $10 \mathrm{~g} / \mathrm{kg} / \mathrm{dia})$, e representar $60 \%$ a $70 \%$ da ingestão energética diária total ${ }^{4}$. Segundo Fogelholm ${ }^{22}$, a exclusão de carne da dieta não prejudica o desempenho em exercícios repetidos de curta duração. Já a redução da ingestão de proteínas e o aumento de carboidratos durante 3 a 5 dias podem resultar em uma melhoria de desempenho em exercício anaeróbico com duração de 2 a 7 minutos. As dietas vegetarianas, freqüentemente, possuem altas taxas de carboidratos, disponibilizando substrato para uma melhor síntese de glicogênio.

Quanto à ingestão protéica, vegetarianos normalmente apresentam valores mais baixos, quando comparados a indivíduos não-vegetarianos $^{18,23}$. Além disso, a qualidade das proteínas de origem vegetal é considerada de baixo valor biológico, visto que são incompletas quanto à composição de aminoácidos. Apesar de necessárias novas investigações, a dieta composta somente por fontes protéicas vegetais parece capaz de satisfazer as necessidades de adultos e crianças saudáveis ${ }^{1}$.

A quota dietética recomendada (RDA) de proteínas é de $0,8 \mathrm{~g} / \mathrm{kg}$ de peso por $\mathrm{dia}^{24}$.
Sabe-se, porém, que atletas de força e endurance necessitam de uma maior ingestão protéica, quando comparados à população saudável sedentária ${ }^{4,25}$. Lemon ${ }^{26}$ sugere que atletas de força, potência ou velocidade aumentem a ingestão para 1,7 a 1,8g/kg/dia, e atletas de endurance para 1,2 a 1,4g/kg/dia. Quantidades superiores não parecem exercer um efeito adicional na performance. Alcançar a adequação de proteínas, em termos quantitativos, a partir de fontes vegetais, é possível, mesmo em uma dieta vegan $^{4,22}$, principalmente quando se faz a combinaçãao de diferentes fontes dietéticas ${ }^{1}$. Ainda assim, há a possibilidade de menor ingestão de aminoácidos essenciais ou mesmo do aminoácido derivado carnitina ${ }^{27}$.

\section{Micronutrientes}

Segundo estudo realizado por Janelle \& $\mathrm{Barr}^{28}$, as mulheres que seguiam dieta vegetariana apresentavam menor ingestão de riboflavina, niacina, vitamina $B_{12}$ e sódio que as não-vegetarianas e maior ingestão de ácido fólico, vitamina $\mathrm{C}$ e cobre. Em pesquisa com jovens vegans de ambos os sexos ${ }^{29}$, foram encontradas baixas ingestões de riboflavina, cobalamina, vitamina $D$, cálcio e selênio. As ingestões de cálcio e selênio permaneceram abaixo do desejável, mesmo após a inclusão de suplementos dietéticos. Pesquisa recente mostrou ingestão deficiente de iodo por indivíduos de ambos os sexos que adotavam dietas vegan e LOV ${ }^{30}$.

O ferro não-heme, proveniente de fontes vegetais e de parte do conteúdo total das carnes, é menos biodisponível que o ferro-heme, encontrado em fontes animais. A eliminação de carnes e o aumento de legumes e grãos integrais na dieta podem resultar na menor ingestão deste mineral, assim como de zinco ${ }^{31}$. Inúmeros efeitos adversos à saúde, vinculados à menor biodisponibilidade de ferro e zinco, têm sido demonstrados pela adoção de dietas vegetarianas. Sabe-se que essa prática alimentar pode, até mesmo, conter mais ferro do que em dietas não vegetarianas, mas o 
mineral encontra-se menos disponível para absorção ${ }^{32}$, dados as diferenças na forma química e os fatores inibidores presentes ${ }^{33,34}$. Apesar dos alimentos vegetais possuírem substâncias que aumentam a captação de ferro, a adoção de dietas vegetarianas precisa ser bem planejada para evitar a deficiência deste mineral ${ }^{23}$. Assim, a recomendação para ingestão de ferro por parte dos vegetarianos é aumentada em $80 \%$ além da RDA ${ }^{32}$. A baixa ingestão de ferro pode levar ao quadro de anemia, interferindo de forma negativa no desempenho, à medida que limita o transporte de oxigênio para os músculos em atividade ${ }^{35}$.

Algumas pesquisas indicam que vegetarianos, especialmente vegans, apresentam ingestão e concentração sérica de vitamina $B_{12}$ (cobalamina) inferiores aos não vegetarianos ${ }^{17,29,36,37}$. Mas, de acordo com Johnston', casos de deficiência desta vitamina são incomuns, mesmo entre os vegans. As razões para tal seriam a baixa necessidade da vitamina, as reservas relativamente grandes e uma circulação enteroepática eficiente, que recupera a maior parte da cobalamina excretada na bile. Ainda assim, a redução na ingestão dessa vitamina tem sido associada, nas dietas vegetarianas (principalmente vegan), ao quadro de hiperhomocisteinemia ${ }^{38,39}$, sendo esse considerado um fator de risco independente para o desenvolvimento de cardiopatias. Janelle \& Barr ${ }^{28}$ sugerem atenção para que quantidades necessárias da vitamina sejam ingeridas. Suplementos orais, ou consumo de produtos enriquecidos com cianocobalamina (forma farmacológica do nutriente), como extrato hidrossolúvel de soja e cereais matinais, são opções que podem auxiliar o atendimento às recomendações.

São menos freqüentes as baixas ingestões de cálcio, zinco, cobalamina e vitamina $D$ entre os lactoovovegetarianos, por consumirem leite e derivados na dieta, fontes significativas desses minerais. Vegans necessitam maior atenção para que não desenvolvam deficiências nutricionais, o que resultaria em efeitos negativos para a saúde e, conseqüentemente, para o desempenho atlético.

\section{Creatina muscular}

A creatina é um composto que contém carbono, hidrogênio e nitrogênio, sintetizada nos rins, pâncreas e fígado (este último principalmente) a partir de três aminoácidos: glicina, arginina e metionina. Apresentando-se na forma fosforilada $(C P)$, atua na refosforilação de ADP (adenosina difosfato), mediante a enzima creatina quinase, mantendo a concentração de ATP (adenosina trifosfato) mais ou menos constante durante esforços de curtíssima duração e alta intensidade. Os estoques intracelulares de creatina total giram em torno de $120-125 \mathrm{mmol} / \mathrm{kg}$ de peso seco, resultando em cerca de $120 \mathrm{~g}$ para um indivíduo de $70 \mathrm{~kg}$, sendo que $95 \%$ desse valor é encontrado nos músculos. Aproximadamente $2 \mathrm{~g}$ (em torno de $1,6 \%$ do total) por dia de creatina é catabolizado em creatinina e excretado pelos rins ${ }^{40}$. A necessidade de creatina é suprida tanto pela ingestão alimentar, quanto pela síntese endógena ${ }^{4,41}$. Em torno de $1 \mathrm{~g}$ é obtido, normalmente, em dietas não-vegetarianas, enquanto que o restante é sintetizado endogenamente.

Especulava-se que indivíduos vegetarianos apresentariam concentração total de creatina menor que aqueles que seguem uma dieta onívora ${ }^{4,41,42}$. Assim, apresentariam melhor resposta à suplementação, devido ao maior aumento de creatina no organismo, pois um maior efeito ergogênico é encontrado nos indivíduos que apresentam, antes da suplementação, baixos níveis musculares do composto ${ }^{4,41}$.

Algumas investigações foram realizadas para elucidar essa questão. Lukaszuk et al. ${ }^{43}$ verificaram que indivíduos que retiraram produtos cárneos da dieta por 21 dias apresentaram diminuição nos níveis musculares de creatina. Entretanto, após a suplementação, seus níveis de creatina não apresentaram diferença significativa em relação aos dos indivíduos que continuaram com o consumo de carnes e também receberam suplementação $\left(148,6 \mathrm{mmol} \mathrm{kg}^{-1}\right.$ e. $141,7 \mathrm{mmol} \mathrm{kg}^{-1}$, respectivamente). Em estudo posterior ${ }^{44}$, por meio de biópsia muscular do músculo vasto lateral, 
foram encontrados níveis mais baixos de creatina total intramuscular nos vegetarianos (117mmol kg-1 contra $130 \mathrm{mmol} \mathrm{kg}^{-1}$ nos onívoros). Além disso, estes apresentaram maiores aumentos nos níveis de fosfocreatina e creatina total e massa muscular, além de melhor desempenho em exercícios de força após a suplementação. Watt et al. ${ }^{45}$ confirmaram esses achados $\left(106,6 \mathrm{mmol} \mathrm{kg}^{-1}\right.$ contra $\left.124,8 \mathrm{mmol} \mathrm{kg}^{-1}\right)$, mas não encontraram diferenças significativas na expressão gênica do transportador de creatina (Creat) entre os grupos, tanto pré como pós-suplementação. Contudo, Shomrat et al. ${ }^{46}$, após a realização de protocolo experimental com vegetarianos e onívoros, verificaram que ambos os grupos apresentaram aumentos similares na potência, após período de suplementação de creatina de uma semana, no teste modificado de Wingate em ciclo-ergômetro.

\section{Alterações fisiológicas}

\section{Alterações hormonais}

A manipulação de variáveis do treinamento, como intensidade, volume e recuperação, pode afetar a resposta hormonal ao exercício. Já a influência da dieta sobre a concentração hormonal é menos compreendida ${ }^{47}$. Raben et al. ${ }^{16}$ verificaram a concentração de testosterona em indivíduos com diferentes dietas. Valores mais baixos foram encontrados em homens que consumiam dieta vegetariana rica em proteína, quando comparados aos que consumiam dieta não-vegetariana, também rica no nutriente. Além disso, os fitoestrógenos da soja, alimento muito consumido por vegetarianos, podem ocasionar concentrações mais baixas de testosterona e androstenediona ${ }^{48}$. Em relação ao IGF-1, um fator de crescimento liberado pelo fígado em resposta ao hormônio do crescimento, estudo realizado com mulheres vegetarianas relata redução em suas concentrações plasmáticas ${ }^{49}$, que pode estar relacionada à baixa ingestão de lipídios, principalmente nos que adotam dieta vegan ${ }^{50,51}$. Os baixos níveis de testosterona sérica e de IGF-1 têm sugerido efeito negativo sobre a hipertrofia muscular e o desenvolvimento da força, visto que são hormônios anabólicos e influenciam na expressão da força, por meio de mecanismos neurais e da síntese protéica ${ }^{52,53}$. Apesar disso, a suplementação protéica à base de vegetal (proteína de soja) mostrou-se capaz de proporcionar um aumento significativamente maior dos níveis de IGF-1, quando comparada à suplementação com proteína do leite, à base de caseína e proteínas do soro ${ }^{54}$.

\section{Função imunológica}

Especulou-se se a dieta vegetariana poderia ocasionar impacto negativo sobre a função imunológica, mas, segundo Fogelholm²2, a retirada da carne da dieta não parece ocasionar efeitos adversos sobre essa função. Quanto à influência da dieta LOV na função imunológica de atletas de endurance, não foram encontradas diferenças significativas na quantidade e atividade das células do sistema imune entre os grupos ${ }^{14}$. Entretanto, sabe-se que o treinamento exaustivo pode causar impacto negativo sobre a função imunológica em atletas, independentemente do tipo de dieta, aumentando a suscetibilidade a infecções, como do trato respiratório superior. Afirma-se que a suplementação de glutamina exerça efeito positivo nesse sentido, atenuando o quadro de imunossupressão pós-exercício ${ }^{21}$.

\section{CONSIDERAÇÕES FINAIS}

De acordo com as publicações científicas atuais, a prática vegetariana pode proporcionar diversos benefícios à saúde humana, desde que bem planejada para atender às necessidades nutricionais. Algumas conclusões puderam ser levantadas, apesar de haver ainda muitas questões a serem elucidadas:

- De acordo com a literatura, não foram encontradas diferenças significativas na capacidade aeróbica e de força em indivíduos que 
seguiram diferentes dietas. As pesquisas que relacionaram o tipo de dieta com o desempenho de força ou atividades supra-máximas são escassas, havendo a necessidade de mais investigações a fim de elucidar a questão.

- Vegetarianos ingerem freqüentemente grandes quantidades de carboidratos, fibras dietéticas, magnésio, potássio, folato, antioxidantes e fitoquímicos. É necessária maior atenção na dieta desses indivíduos, no que diz respeito à ingestão de cálcio, zinco, ferro e vitamina $B_{12}$. A ocorrência de anemia por deficiência de ferro pode ser mais comum na mulher atleta, o que interfere negativamente no rendimento. $A$ baixa ingestão de vitamina $B_{12}$ pode levar ao quadro de hiperhomocisteinemia, aumentando o risco de desenvolvimento de doenças cardiovasculares.

- O conteúdo protéico dos alimentos de origem vegetal é freqüentemente menor, além de apresentarem menor valor biológico, pois possuem aminoácidos limitantes. Ainda assim, os pesquisadores sugerem que a ingestão protéica nas dietas vegetarianas pode cumprir a cota de fornecimento adequada, mesmo para atletas que necessitam de maior ingestão protéica.

- Uma dieta vegetariana necessita ser bem planejada e equilibrada em termos nutricionais, sendo, assim, apropriada a todos os estágios do desenvolvimento humano, incluindo a gestação, lactação, infância e adolescência, além de proporcionar suporte adequado ao desempenho esportivo.

- Os níveis de creatina intramusculares dos vegetarianos são mais baixos, o que pode afetar o rendimento em exercícios supramáximos. Em contrapartida, a suplementação de creatina monohidratada pode proporcionar maior efeito ergogênico nesses indivíduos.

- Vegans e vegetarianos podem apresentar níveis mais baixos de hormônios anabólicos, como a testosterona, androstenediona e IGF-1, o que, talvez, interfira diretamente no desenvolvimento da força e hipertrofia musculares. A função imunológica não parece ser afetada.
Embora com qualidade nutricional diferente da onívora, a dieta vegetariana, desde que supra as adequações nutricionais do atleta, não prejudica o seu desempenho aeróbio. No que se refere ao desempenho hipertrófico ou de força e potência muscular, os resultados não são conclusivos.

\section{REFERÊ N CIAS}

1. Johnston PK. Implicações nutricionais das dietas vegetarianas. In Shils ME, Olson JA, Shike M, Ross AC. Tratado de nutrição moderna na saúde e na doença. 9.ed. São Paulo: Manole; 2003. p.1885-99.

2. Soares, EA, Burini, RC. Dietas vegetarianas: tipos, origem e implicações nutricionais. Cad Nutr. 1990; 1:3-18.

3. Jenkins $D J$, Kendall $C W$, Marchie $A$, Jenkins $A L$, Augustin LS, Ludwig DS, et al. Type 2 diabetes and the vegetarian diet. Am J Clin Nutr. 2003; 78(3 Suppl):610S-6.

4. Nieman DC. Physical fitness and vegetarian diets: is there a relation? Am J Clin Nutr. 1999: 70(3 Suppl):570S-5S

5. Szeto YT, Kwok TC, Benzie IF. Effects of a long-term vegetarian diet on biomarkers of antioxidant status and cardiovascular disease risk. Nutrition. 2004; 20(10):863-6.

6. Singh PN, Sabate J, Fraser GE. Does low meat consumption increase life expectancy in humans? Am J Clin Nutr. 2003; 78(3 Suppl):526S-32S.

7. American Dietetic Association, Dietitians of Canada. Position of the American Dietetic Association and Dietitians of Canada: vegetarian diets. Can J Diet Pract Res. 2003; 64(2):62-81.

8. Richter V, Rassoul F, Hentschel B, Kothe K, Krobara $M$, Unger R, et al. Age-dependence of lipid parameters in the general population and vegetarians. Z Gerontol Geriatr. 2004; 37(3): 207-13.

9. Sabate J. The contribution of vegetarian diets to health and disease: a paradigm shift? Am J Clin Nutr. 2003; 78(3 Suppl):502S-7S.

10. Cotes JE, Dabbs JM, Hall AM. Possible effect of vegan diet upon lung function and the cardiorespiratory response to submaximal exercise in healthy women. J Physiol. 1970; 1(209):30-2.

11. Hanne N, Dlin R, Rotstein A. Physical Fitness, anthropometric and metabolic parameters in 
vegetarian athletes. J Sports Med Phys Fitness. 1986; 26(2):180-5.

12. Nieman DC, Haig JL, De Guia ED, Dizon GP, Register UD. Reducing diet and exercise training effects on resting metabolic rates in mildly obese women. $J$ Sports Med Phys Fitness. 1988; 28(1):79-88.

13. Nieman DC, Sherman KM, Arabatzis K, Underwood BC, Barbosa JC, Johnson M, et al. Hematological, anthropometric, and metabolic comparisons between vegetarian and nonvegetarian elderly women. Int J Sports Med. 1989; 10(4):243-51.

14. Snyder AC, Dvorak LL, Roepke JB. Influence of dietary iron source on measures of iron status among female runners. Med Sci Sports Exerc. 1989; 21(1):7-10.

15. Richter EA, Kiens B, Raben A, Tvede N, Pedersen BK. Immune parameters in male athletes after a lacto-ovo-vegetarian diet and a mixed Western diet. Med Sci Sports Med. 1991; 23(5):517-21.

16. Raben A, Kiens B, Richter EA, Rasmussen LB, Svenstrup B, Micic $S$, et al. Serum sex hormones and endurance performance after a lacto-ovo vegetarian and a mixed diet. Med Sci Sports Exerc. 1992; 24(11):1290-7.

17. Eisinger M, Plath $M$, Jung K, Leitzmann C. Nutrient intake of endurance runners with ovo-lactovegetarian diet and regular western diet. Zeitschrift fur Ernahrungswiss. 1994; 33(3):217-29.

18. Hebbelinck M, Clarys P, Malsche AD. Growth, development, and physical fitness of Flemish vegetarian children, adolescents, and young adults. Am J Clin Nutr. 1999; 70(3 Suppl):579S-85S.

19. Campbell WW, Barton ML, Cyr-Campbell D, Davey $\mathrm{SL}$, Beard JL, Parise G, et al. Effects of an omnivorous diet compared with a lactoovovegetarian diet on resistance-training-induced changes in body composition and skeletal muscle in older men. Am J Clin Nutr. 1999; 70(3):1032-9.

20. American College of Sports Medicine. Nutrition and athletic performance. Med Sci Sports Exerc. 2000; 32(12):2130-45.

21. Williams MH. Nutrição para saúde, condicionamento físico e desempenho esportivo. São Paulo: Manole; 2002.

22. Fogelholm, M. Dairy products, meat and sports performance. Sports Med. 2003; 33(8):615-31

23. Ball MJ, Bartlett MA. Dietary intake and iron status of Australian vegetarian women. Am J Clin Nutr. 1999; 70(3):353-8.

24. Food and Nutrition Board, Institute of Medicine. Dietary Reference Intakes for Energy, Carbohydrate, Fiber, Fat, Fatty Acids, Cholesterol, Protein, and Amino Acids (Macronutrients). Washington (DC): National Academy Press; 2002.
25. Tarnopolsky MA. Protein and physical performance. Curr Opin Clin Nutr Metab Care. 1999; 2(6):533-7.

26. Lemon P. Dietary protein requeriments in athletes. J Nutr Biochem. 1997; 28:52-60.

27. Stanley CA. Carnitine Deficiency Disorders in Children. Ann N Y Acad Sci. 2004; 1033:42-51.

28. Janelle KC, Barr SI. Nutrient intakes and eating behavior scores of vegetarian and nonvegetarian women. J Am Diet Assoc. 1995; 95(2):180-6.

29. Larsson $C L$, Johansson GK. Dietary intake and nutritional status of young vegans and omnivores in Sweden. Am J Clin Nutr. 2002; 76(1):100-6.

30. Krajcovicova-Kudlackova M, Buckova K, Klimes I, Sebokova E. lodine deficiency in vegetarians and vegans. Ann Nutr Metab. 2003; 47(5):183-5.

31. Hunt JR. Bioavailability of iron, zinc, and other trace minerals from vegetarian diets. Am J Clin Nutr. 2003; 78(3):633S-9S.

32. Food and Nutrition Board, Institute of Medicine. Dietary reference intakes for vitamin A, vitamin K, arsenic, boron, chromium, copper, iodine, iron, manganese, molybdenium, nickel, silicon, vanadium, and zinc. Washington (DC): National Academy Press; 2000.

33. Hunt JR, Roughead ZK. Nonheme-iron absorption, fecal ferritin excretion, and blood indexes of iron status in women consuming controlled lactoovovegetarian diets for 8 weeks Am J Clin Nutr. 1999; 69(5):944-52.

34. Hunt JR, Vanderpool RA. Apparent copper absorption from a vegetarian diet. Am J Clin Nutr. 2001; 74(6):803-7.

35. Gledhill N, Warburton D, Jamnik V. Haemoglobin, blood volume, cardiac function, and aerobic power. Can J Appl Physiol. 1999; 24(1):54-65.

36. Huang YC, Chang SJ, Chiu YT, Chang HH, Cheng $\mathrm{CH}$. The status of plasma homocysteine and related B-vitamins in healthy young vegetarians and nonvegetarians. Eur J Nutr. 2003; 42(2):84-90.

37. Hokin BD, Butler T. Cyanocobalamin (vitamin B12) status in Seventh-day Adventist ministers in Australia. Am J Clin Nutr. 1999; 70(3 Suppl): 576S-8.

38. Herrmann W, Schorr H, Obeid R, Geisel J. Vitamin B-12 status, particularly holotranscobalamin II and methylmalonic acid concentrations, and hyperhomocysteinemia in vegetarians. Am J Clin Nutr. 2003; 78(1):131-6.

39. Stabler SP, Allen RH. Vitamin B12 deficiency as a worldwide problem. Annu Rev Nutr. 2004; 24:299-326. 
40. Greenhaff PL. The nutritional biochemistry of creatine. Nutr Biochem. 1997; 8:610-8.

41. Williams MH, Kreider RB, Branch JD. Creatina. São Paulo: Manole; 2000.

42. Maughan RJ. Creatine supplementation and exercise performance. Int J Sport Nutr. 1995; 5(2):100-10.

43. Lukaszuk JM, Robertson RJ, Arch JE, Moore GE, Yaw Km, Kelley DE, et al. Effect of creatine supplementation and a lacto-ovo-vegetarian diet on muscle creatine concentration. Int J Sport Nutr Exerc Metab. 2002; 12(3):336-48.

44. Burke DG, Chilibeck PD, Parise G, Candow DG, Mahoney D, Tarnopolsky M. Effect of creatine and weight training on muscle creatine and performance in vegetarians. Med Sci Sports Exerc. 2003; 35(11):1946-55.

45. Watt KK, Garnham AP, Snow RJ. Skeletal muscle total creatine content and creatine transporter gene expression in vegetarians prior to and following creatine supplementation. Int J Sport Nutr Exerc Metab. 2004; 14(5):517-31.

46. Shomrat A, Weinstein $Y$, Katz A. Effect of creatine feeding on maximal exercise performance in vegetarians. Eur J Appl Physiol. 2000; 82(4): 321-5.

47. Volek JS, Kraemer WJ, Bush JA, Incledon T, Boetes $M$. Testosterone and cortisol in relationship to dietary nutrients and resistance exercise. J Appl Physiol. 82(1):49-54

48. Weber KS, Setchell KD, Stocco DM, Lephart ED. Dietary soy-phytoestrogens decrease testosterone levels and prostate weight without altering $\mathrm{LH}$, prostate 5alpha-reductase or testicular steroidogenic acute regulatory peptide levels in adult male Sprague-Dawley rats. J Endocrinol. 2001; 170(3):591-9.

49. Allen NE, Appleby PN, Davey GK, Kaaks R, Rinaldi $S$, Key TJ. The associations of diet with serum insulin-like growth factor I and its main binding proteins in 292 women meat-eaters, vegetarians, and vegans. Cancer Epidemiol Biomarkers Prev. 2002; 11(11):1441-8

50. McCarty MF. A low-fat, whole-food vegan diet, as well as other strategies that down-regulate IGF-I activity, may slow the human aging process. Med Hypotheses. 2003a; 60(6):784-92.

51. McCarty MF. IGF-I activity may be a key determinant of stroke risk: a cautionary lesson for vegans. Med Hypotheses. 2003b; 61(3):323-34.

52. Palo EF, Gatti R, Lancerin F, Cappellin E, Spinella P. Correlations of growth hormone $(\mathrm{GH})$ and insulin-like growth factor I (IGF-I): effects of exercise and abuse by athletes. Clin Chim Acta. 2001: 305(1-2):1-17.

53. Hartgens F, Kuipers H. Effects of androgenic-anabolic steroids in athletes. Sports Med. 2004; 34(8):513-54.

54. Khalil DA, Lucas EA, Juma S, Smith BJ, Payton ME, Arjmandi BH. Soy protein supplementation increases serum insulin-like growth factor-I in young and old men but does not affect markers of bone metabolism. J Nutr. 2002; 132(9):2605-8.

Recebido em: 19/7/2005

Versão final reapresentada em: 1/2/2006 Aprovado em: 19/4/2006 
